\title{
Improving pre-salt image with reflection full-waveform inversion in Santos Basin
}

\author{
Wei Kang, Marcela Ortin, Joel Monteiro, Marina Pantoja, Olga Zdraveva, Denes Vigh, WesternGeco
}

Copyright 2019, SBGf - Sociedade Brasileira de Geofísica

This paper was prepared for presentation during the $16^{\text {th }}$ International Congress of the Brazilian Geophysical Society held in Rio de Janeiro, Brazil, 19-22 August 2019.

Contents of this paper were reviewed by the Technical Committee of the $16^{\text {th }}$ International Congress of the Brazilian Geophysical Society and do not necessarily represent any position of the SBGf, its officers or members. Electronic reproduction or storage of any part of this paper for commercial purposes without the written consent of the Brazilian Geophysical Society is prohibited.

\section{Abstract}

Over the last ten years, full-waveform inversion (FWI) has become established as an integral part of earth model building (EMB) workflows. Recently, the industry witnessed the introduction of many types of FWI, differing either by the portion of the wavefield used in the inversion or by the nature of the objective function. Among these methods, reflection full-waveform inversion (RFWI) can utilize reflection data to build smooth velocity updates that reach beyond the penetration depth of refractions that are used in conventional FWI. Due to the limited opening angle of subsalt illumination, RFWI becomes more and more popular to update velocity models in the subsalt zone. In this case study in the Santos Basin, we discuss RFWI strategies and results, in which the pre-salt target level image has a better quality and continuity after being migrated with a more-detailed model created using a comprehensive EMB workflow that includes common image point tomography and conventional least-squares FWI for the post-salt, CIP tomography and RFWI for the salt and pre-salt. From the various existing RFWI strategies, we adopted Born modelling to generate reflections and a traveltime-based adjustive objective function, and then form the gradient with a combination of crosscorrelation between forward or backward and source or scattered wavefield. This approach will be validated with quality controls in both the shot and image domains. The updated model along with its updated image generate modelled shots that are more consistent with observed data, and the updated image is more geologically plausible with stronger focusing and continuous events.

\section{Introduction}

The introduction of full-waveform inversion (FWI) as a production-scale model-updating tool almost ten years ago (Vigh and Starr, 2008) led to the corresponding modifications of the earth model building (EMB) workflows used throughout the industry. By 2011, case studies involving real data examples started to show some promise, but the widespread use in different geologic environments, and with different types of field data, was triggered by the appearance of the FWI tools less prone to cycle skipping that relaxed the requirements for very accurate starting models and/or ultra-low frequencies and long offsets such as described by Warner and Guasch
(2014) and Jiao et al. (2015). Most recent developments are related to a more-focussed use of reflected rather than transmitted energy and were enabled by the ability to separate the high- and low-wavenumber components of the reflection data terms in the FWI gradient (Sun et al., 2016). Today, this type of FWI is often referred to as reflection FWI (RFWI) and most successful applications are based on a kinematics-driven objective function (Sun et al., 2018).

In the past, CIP tomography (Woodward et al. 2008) was the main tool used in the inversion stage of the anisotropic EMB workflows (Zdraveva et al., 2012), while today, it is often alternating with different types of FWI. Zhang et al. (2017) and Yanez et al. (2018) demonstrated successful application of a workflow based on adjustive FWI (AdjFWI) (Jiao et al., 2015), followed by conventional LS FWI to resolve shallow fluvial channels in the North Sea, while reducing turnaround time (TAT) with the parallel execution with signal processing and data conditioning for multi-layer common image point (CIP) tomography. Menzel-Jones et al. (2018) demonstrated similar TAT advantages, while resolving shallow gas anomalies with a combination of AdjFWI and least-squares (LS) FWI. In deep water, complex geology, and subsalt environments, where the use of transmitted energy is often limited by the finiteacquisition spread, the use of RFWI gained popularity.

Herein, we show the application of our RFWI strategy on narrow-azimuth data acquired in the Santos Basin deepwater area. The Santos Basin is a large sedimentary basin (around 400,000 km²) located offshore southeast Brazil. The Lithology of the Santos Basin has mixed sequences of carbonates and silicates intercalated with volcanic rocks. One of its characteristics is that it is strongly stratified with complex salt bodies and evaporites (Moreira et al., 2007). These complex features can be a real challenge when it comes to imaging and data processing. The Santos Basin is located on the southeast portion of Brazilian margin, in front of the south littoral of Rio de Janeiro, São Paulo, Paraná, and north of Santa Catarina. Geologically, it is a depression limited to the north by the Cabo Frio elevation and to the south by the Florianopolis elevation. This basin covers approximately $350,000 \mathrm{~km}^{2}$, of which $200,000 \mathrm{~km}^{2}$ are located under up to $40 \mathrm{~m}$ of water and $150,000 \mathrm{~km}^{2}$ between $400 \mathrm{~m}$ and $3,000 \mathrm{~m}$. In this region, post-salt reservoirs have been discovered for decades, and pre-salt has become the target in recent years. However, complex geology makes pre-salt imaging quite challenging. Even if the post-salt model has been correctly established, variant salt velocity with evaporite and halite plus irregular salt geometry remain problematic to model building and imaging. Besides, the pre-salt model building also suffers from a limited opening angle due to limited cable length and azimuth of data acquisition. 


\section{Method}

Full-waveform inversion measures the difference between predicted and observed seismic data and updates the model by solving a non-linear optimization problem:

$$
\min _{\mathbf{m}} \frac{1}{2}\left\|\mathbf{d}_{p r d}[\mathbf{m}]-\mathbf{d}_{o b s}\right\|^{2}
$$

where $\mathbf{m}$ stands for normally the seismic velocity model and $\mathbf{d}_{\text {prd }}$ and $\mathbf{d}_{\text {obs }}$ denote predicted and observed shot records. It is well discussed that simply using a fullwavefield difference with a kinematically inaccurate starting model will cause the cycle-skipping issue and make the inversion fail to converge. This leads to two effective types of conventional full-waveform inversion. The first type is to input a model with good kinematics and then target at adding high-wavenumber details into the starting model. The second type uses the refraction part of data, which is more linearly related to the velocity model, combined with a traveltime-based objective function to achieve a low-wavenumber component update. Due to limited receiver cable length, refractions normally only penetrate the shallow part of the model depending on maximum offset, water depth, and velocity gradient of real earth.

RFWI can utilize the reflection part of data to improve the model beyond the penetration depth of refraction data with low-wavenumber background velocity update. Considering a migration impulse response (Figure 1), it can be decomposed to three components: the elliptical migrationstyle high-wavenumber event, the shallow 'bananashaped' low-wavenumber part, and the 'rabbit-ear-shaped' low wavenumber energy that is normally referred to as backscattered noise in reverse-time migration (RTM). The first two components correspond to the two types of conventional FWI mentioned above, and the third component is what we are pursuing to extract to form RFWI gradient. Three important components of the RFWI strategy need to be addressed: modelling reflections with a smooth model, objective function and extracting low wave-number gradient. Among existing strategies, we adopted born modelling to generate reflections and a travel-time based adjustive objective function, and then form the gradient with combined cross-correlation between forward or backward and source or scattered wavefield.

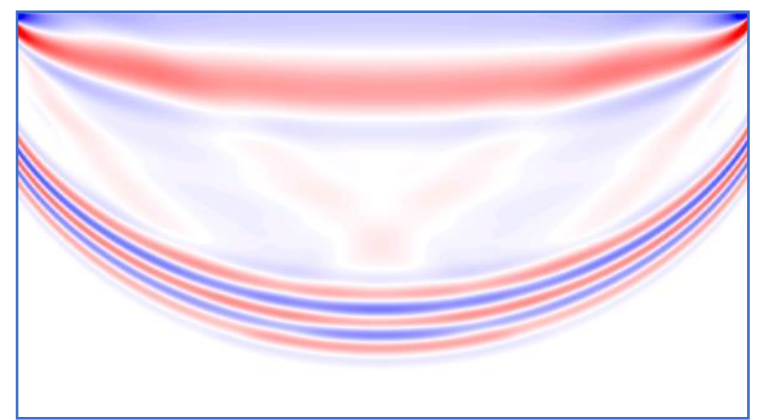

Figure 1 - Illustration of single trace impulse response with cross-correlation of source and receiver wavefield. It contains three types of FWI gradient.

\section{Results}

In this section, we will demonstrate the application of RFWI on field data to improve pre-salt imaging. Narrow-azimuth data with noise attenuation, de-ghost, and de-multiple process is used as input data for RFWI. Post-salt velocity model has been built with an integrated earth model building workflow including high-resolution tomography and conventional least-square FWI. Salt and pre-salt velocity has been updated with velocity scanning and intrasalt tomography on RTM subsurface azimuth angle gathers (SAAGs) (Vyas et al, 2011).

RFWI was conducted at frequency band of $2-11 \mathrm{~Hz}$. First a wavelet was extracted from the filtered shot data using the water bottom reflection. Wavelets extracted from each individual shot were consistent and the stacked average wavelet is shown in Figure 2. For computational efficiency, in every iteration a regular RTM was run to generate reflectivity for modelling reflections in Born modelling instead of least-square migration. Basic image enhancement was then applied to further improve coherency of reflectivity. Since the target updating zones are salt and pre-salt, post-salt image has been muted to exclude their impact on the gradient. Comparison between observed shot and modelled reflections below top of salt with models before and after RFWI are displayed in Figure 3. Reflections modelled with the updated model (Figure 3b) show better consistency with observed data compared to the input model (Figure 3a), which validated that reflection FWI managed to match correct reflection events by optimizing the travel-time objective function.

The improvement of data matching in the shot domain benefited the pre-salt migration in the image domain. Figure 4 and Figure 5 show the RFWI model update and image uplift on a depth slice and inline profile respectively. RFWI slowed down the evaporate area inside salt and high pore-pressure zone in the pre-salt, which matched our geology and rock physics understanding. This velocity update brought important uplift on pre-salt imaging with more continuous and focused reflection events. The model update was also validated on RTM SAAGs in Figure 6. Gather locations are indicated in the yellow box in Figure 5c. RFWI updated SAAGs show stronger focusing and gather flatness in the pre-salt region, which also provided a more reliable input for subsequent pre-salt angle-gather tomography.

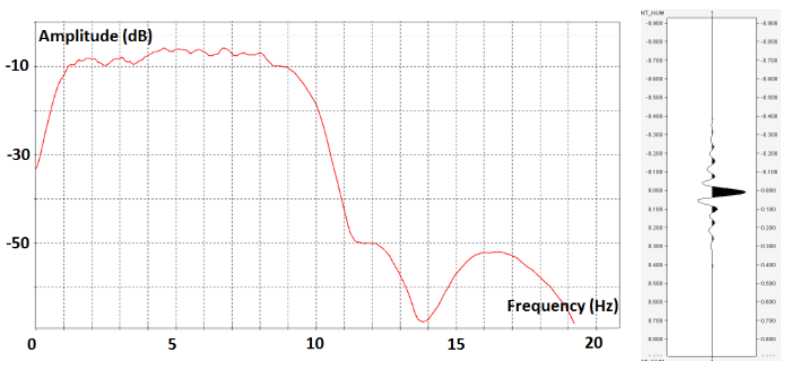

Figure 2 - Frequency spectrum of filtered data used in inversion (left) and its extracted wavelet (right). 

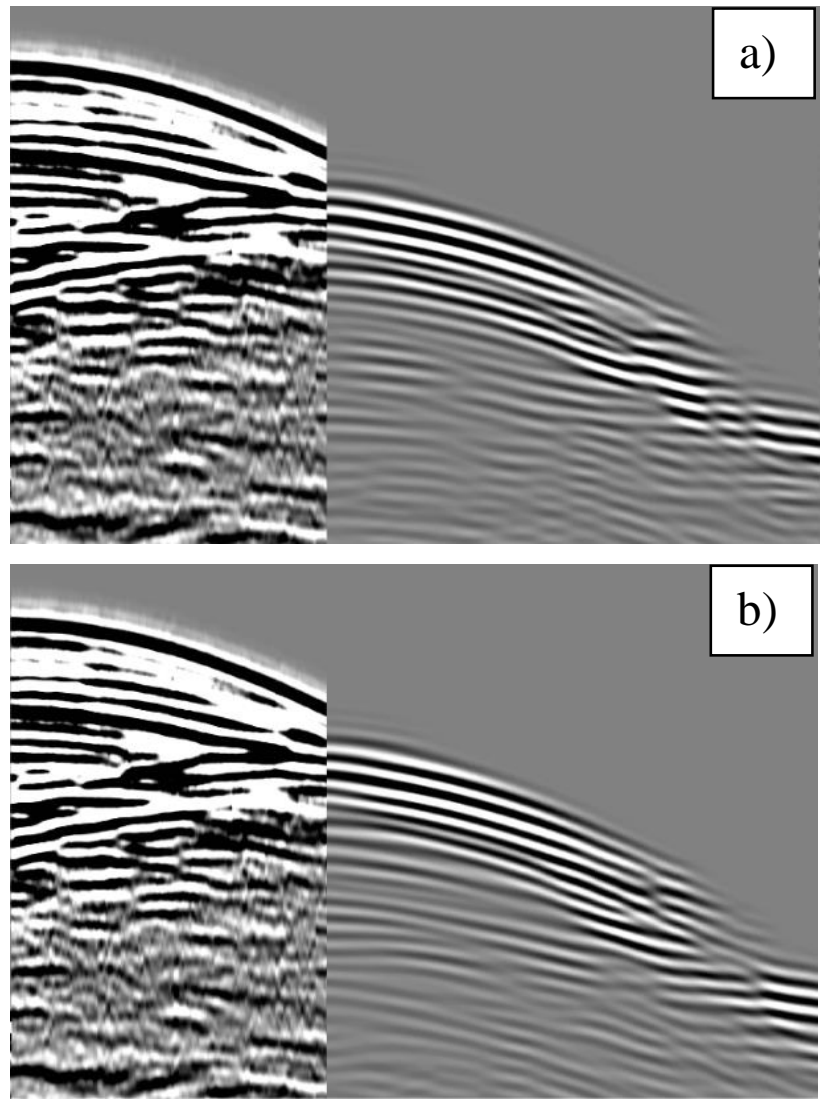

Figure 3 - Comparison between observed (left) and predicted (right) shot record. a) Using input model and its corresponding image, b) using RFWI model and its corresponding image. Missing events close to the water bottom reflection were caused by the mute on input reflectivity for Born modelling. Reflections modelled with updated velocity and its corresponding image match the observed data better in salt and pre-salt part.

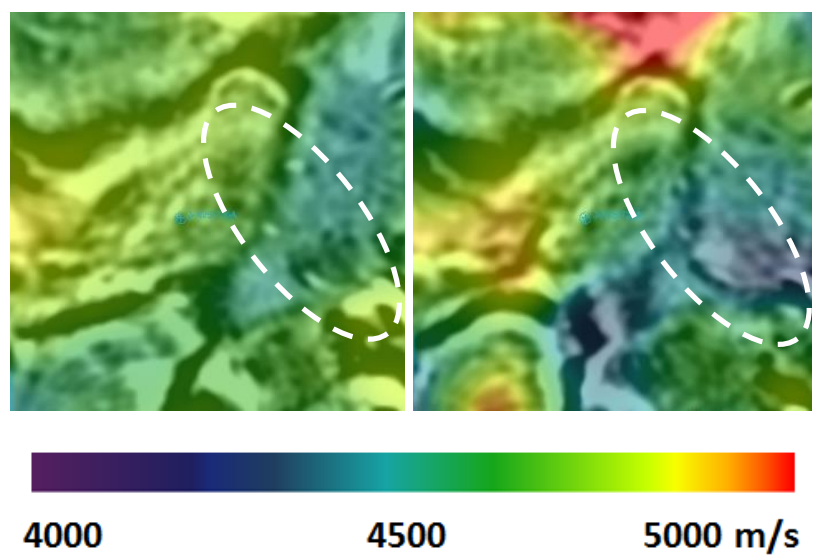

Figure 4 - Input (left) and updated (right) velocity model overlaid on its corresponding RTM image depth slice in the pre-salt zone.
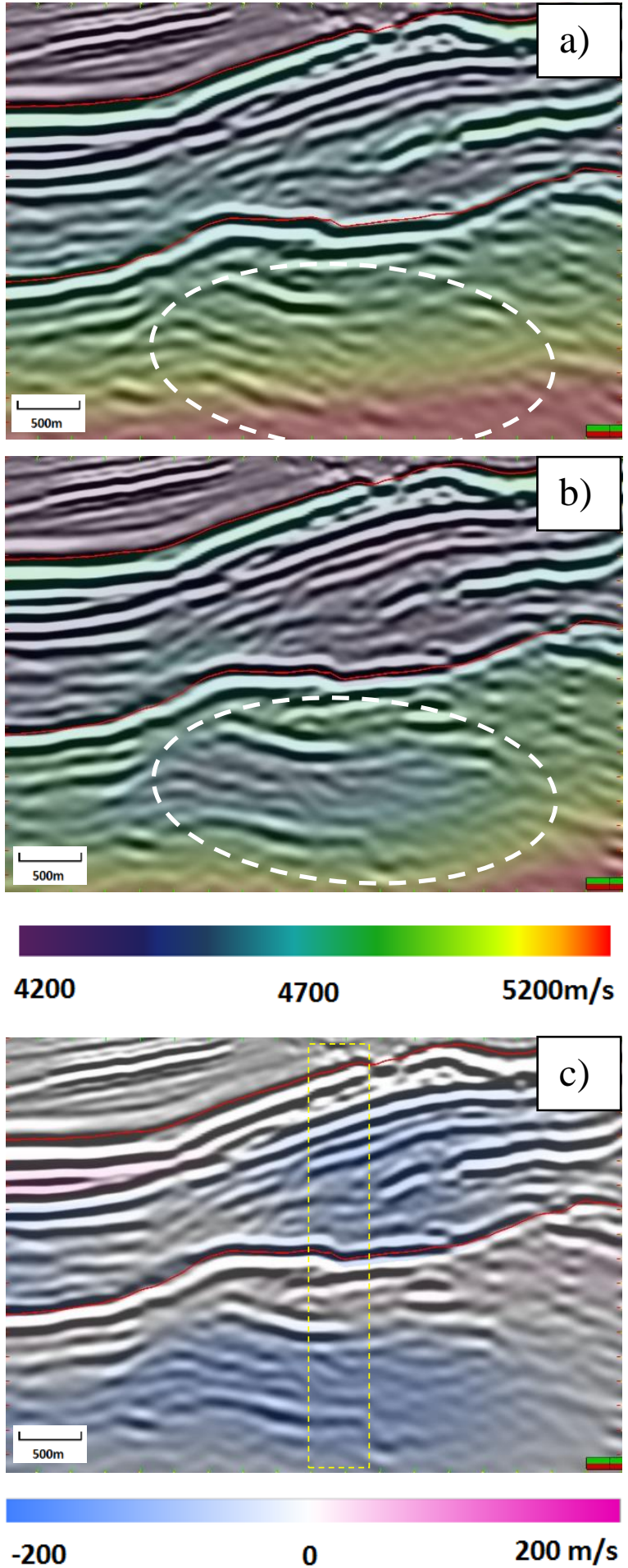

Figure 5 - Model and image update quality control between input and RFWI. a) Input velocity model and its corresponding RTM image, b) RFWI updated velocity model and its corresponding RTM image, c) RFWI velocity update overlaid on updated image. RTM was run at maximum frequency of $18 \mathrm{~Hz}$ with no image enhancement applied. 


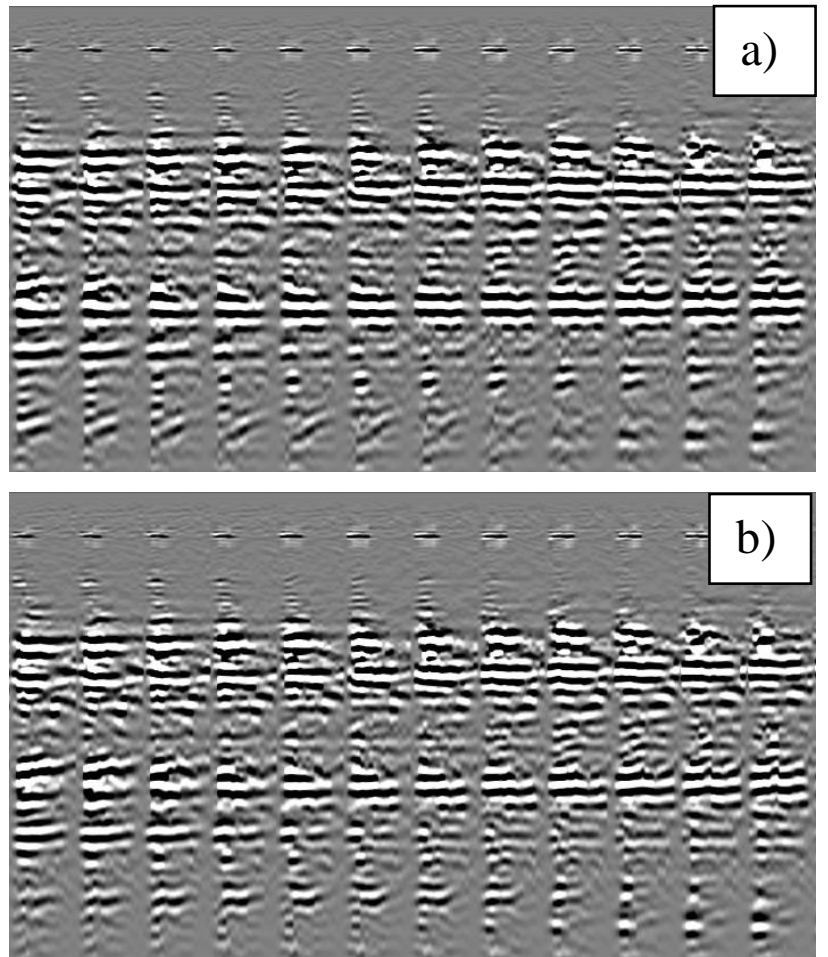

Figure 6 - RTM SAAGs comparison between: a) input model and b) RFWI updated model. (Location indicated by box in Figure $5 \mathrm{c}$ )

\section{Conclusions}

In this project we applied an integrated EMB workflow to achieve the goal of a better pre-salt image. A key tool in this process was the RFWI strategy adopted, including; born modelling to generate reflections plus travel-time based adjustive objective function, and then form the gradient with combination of cross-correlation between forward or backward and source or scattered wavefield. The field data example in Santos Basin showed successful application of our RFWI strategy. In the shot domain, the updated model shows better matching for reflections between observed and modelled data; and in the image domain, the migrated image using the updated model could generate more continuous and geologically plausible events, which was also validated with the RTM subsurface azimuth angle gathers.

\section{Acknowledgments}

We thank Petrobras for permission to present this work and close interaction with WesternGeco. We also thank WesternGeco for permission and support to this project.
JIAO, K., SUN, D., CHENG, X. AND VIGH, D. Adjustive full waveform inversion. 85th Annual International Meeting, SEG, Expanded Abstracts, p. 1091-1095, 2015.

MENZEL-JONES, G., THAM, M., SAZYKIN, A., CHEAH, W.L., KRISTIANSEN, P., GOH, V., VADER, H., NAYAK, P. AND GERRITSEN, S. Uncovering the missing data in the gas cloud with $\mathrm{P}-\mathrm{P}$ waves imaging: the first deepwater OBN survey from Southeast Asia. 88th Annual International Meeting, SEG, Expanded Abstracts, p. 41364140, 2018.

MOREIRA, J.L.P., MADEIRA, C.V., GIL, J.A., MACHADO, M.A.P. Bacia de Santos. Boletim de Geociências da Petrobras v. 15, n. 2, p. 531-549, 2007.

SUN, D., JIAO, K., CHENG, X., AND VIGH, D. Reflectionbased waveform inversion. 86th Annual International Meeting, SEG, Expanded Abstracts, p. 1151-1156, 2016.

SUN, D., JIAO, K., CHENG, X., AND VIGH, D. Keys to robust reflection based full waveform inversion, 88th Annual International Meeting, SEG, Expanded Abstracts, p. 1283-1287, 2018.

VIGH, D. AND STARR, B. 3D prestack plane-wave fullwaveform inversion. Geophysics, 73, 5, VE135-VE144, 2008.

VYAS, M., DU, X., MOBLEY, E. AND FLETCHER, R., Methods for computing angle gathers using RTM: $72^{\text {nd }}$ EAGE Conference and Exhibition, Extended Abstracts, 2011

WARNER, M. AND GUASCH, L. Adaptive waveform inversion theory. 84th Annual International Meeting, SEG, Expanded Abstracts, p. 1089-1093, 2014.

Woodward, M., Nichols, D., Zdraveva, O., Whitfield, P. and Johns, T. A decade of tomography. Geophysics, 73, 5, VE5-VE11, 2008.

YANEZ, M., TYAGI, S., STEIGER-JARVIS, M., BAILEY, J., SHADRINA. M. AND DINGWALL, S. Addressing the challenges heritage surface seismic data in a complex shallow water environment for improved overburden imaging and reservoir characterization. 88th Annual International Meeting, SEG, Expanded Abstracts, p. 11231127, 2018.

ZDRAVEVA, O., HUBBARD, R., O'BRIAIN, M., ZHANG, D. AND VITO, C. Anisotropic model building in complex media: Three successful strategies applied to wide azimuth data from Gulf of Mexico. 74th EAGE Conference and Exhibition, Extended Abstracts, 2012.

ZHANG, L., ZIMMERMANN, N., BULLOCK, A., KHLUSSOVICH, T., RUNDE, Ø. AND SKORVE, T. Anisotropic earth model building using multi-survey full waveform inversion with ocean bottom cable and streamer datasets. 79th EAGE Conference and Exhibition, 2017.

\section{References}

